\title{
THE LEAST H-EIGENVALUE OF ADJACENCY TENSOR OF HYPERGRAPHS WITH CUT VERTICES
}

\author{
YI-ZHENG FAN*, ZHU ZHU, AND YI WANG
}

\begin{abstract}
Let $G$ be a connected hypergraph with even uniformity, which contains cut vertices. Then $G$ is the coalescence of two nontrivial connected sub-hypergraphs (called branches) at a cut vertex. Let $\mathcal{A}(G)$ be the adjacency tensor of $G$. The least H-eigenvalue of $\mathcal{A}(G)$ refers to the least real eigenvalue of $\mathcal{A}(G)$ associated with a real eigenvector. In this paper we obtain a perturbation result on the least H-eigenvalue of $\mathcal{A}(G)$ when a branch of $G$ attached at one vertex is relocated to another vertex, and characterize the unique hypergraph whose least $\mathrm{H}$-eigenvalue attains the minimum among all hypergraphs in a certain class of hypergraphs which contain a fixed connected hypergraph.
\end{abstract}

\section{INTRODUCTION}

Recently, the spectral hypergraph theory developed rapidly, the adjacency tensors $\mathcal{A}(G)$ [ of uniform hypergraphs $G$ were introduced to investigating the structure of hypergraphs, just like adjacency matrices to simple graphs. As $\mathcal{A}(G)$ is nonnegative, by using Perron-Frobenius theorem 2, 11, 22, 23, 24, many results about its spectral radius are presented [3, 4, 5, 8, 16, 18.

For the least H-eigenvalue of $\mathcal{A}(G)$ of a $k$-uniform connected hypergraph $G$, Khan and Fan [15] discussed the limit points of the least H-eigenvalue of $\mathcal{A}(G)$ when $G$ is non-odd-bipartite. Let $\rho(G)$ be the spectral radius of $\mathcal{A}(G)$. Shao et al. 21] proved that the $-\rho(G)$ is an H-eigenvalue of $\mathcal{A}(G)$ if and only if $k$ is even and $G$ is odd-bipartite. Some other equivalent conditions are summarized in 9 . Note that $-\rho(G)$ is an eigenvalue of $\mathcal{A}(G)$ if and only if $k$ is even and $G$ is odd-colorable $[9]$. So, there exist odd-colorable but non-odd-bipartite hypergraphs [7, 19, for which $-\rho(G)$ is an N-eigenvalue. An odd-colorable hypergraph has a symmetric spectrum. One can refer 6 for more results on the spectral symmetry of nonnegative tensors and hypergraphs.

To our knowledge, the least H-eigenvalue of $\mathcal{A}(G)$ receives little attention except the above work. If $k$ is even, then the least H-eigenvalue of $\mathcal{A}(G)$ is a solution of minimum problem over a real unit sphere; see Eq. (2.3). So, throughout of this paper, when discussing the least H-eigenvalue of $\mathcal{A}(G)$, we always assume that $G$ is connected with even uniformity $k$. For convenience, the least H-eigenvalue of $\mathcal{A}(G)$ is simply called the least eigenvalue of $G$ and the corresponding H-eigenvectors are called the first eigenvectors of $G$.

2010 Mathematics Subject Classification. Primary 15A18, 05C65; Secondary 13P15, 14M99.

Key words and phrases. Hypergraph, adjacency tensor, least H-eigenvalue, eigenvector, perturbation.

*The corresponding author. This work was supported by National Natural Science Foundation of China (Grant No. 11871073, 11771016). 
In this paper we give a perturbation result on the least H-eigenvalue of $\mathcal{A}(G)$ when a branch of $G$ attached at one vertex is relocated to another vertex, and characterize the unique hypergraph whose least $\mathrm{H}$-eigenvalue attains the minimum among all hypergraphs in a certain class of hypergraphs which contain a fixed connected hypergraph. The perturbation result in this paper is a generalization of that on the least eigenvalue of the adjacency matrix of a simple graph in [10].

\section{Preliminaries}

2.1. Tensors and eigenvalues. A real tensor (also called hypermatrix) $\mathcal{A}=$ $\left(a_{i_{1} i_{2} \ldots i_{k}}\right)$ of order $k$ and dimension $n$ refers to a multi-dimensional array with entries $a_{i_{1} i_{2} \ldots i_{k}} \in \mathbb{R}$ for all $i_{j} \in[n]:=\{1,2, \ldots, n\}$ and $j \in[k]$. Clearly, if $k=2$, then $\mathcal{A}$ is a square matrix of dimension $n$. The tensor $\mathcal{A}$ is called symmetric if its entries are invariant under any permutation of their indices.

Given a vector $x \in \mathbb{C}^{n}, \mathcal{A} x^{k} \in \mathbb{C}$ and $\mathcal{A} x^{k-1} \in \mathbb{C}^{n}$, which are defined as follows:

$$
\begin{aligned}
\mathcal{A} x^{k} & =\sum_{i_{1}, i_{2}, \ldots, i_{k} \in[n]} a_{i_{1} i_{2} \ldots i_{k}} x_{i_{1}} x_{i_{2}} \cdots x_{i_{k}}, \\
\left(\mathcal{A} x^{k-1}\right)_{i} & =\sum_{i_{2}, \ldots, i_{k} \in[n]} a_{i i_{2} \ldots i_{k}} x_{i_{2}} \cdots x_{i_{k}}, i \in[n] .
\end{aligned}
$$

Let $\mathcal{I}=\left(i_{i_{1} i_{2} \ldots i_{k}}\right)$ be the identity tensor of order $k$ and dimension $n$, that is, $i_{i_{1} i_{2} \ldots i_{k}}=1$ if $i_{1}=i_{2}=\cdots=i_{k} \in[n]$ and $i_{i_{1} i_{2} \ldots i_{k}}=0$ otherwise.

Definition 2.1 ([17, 20]). Let $\mathcal{A}$ be a real tensor of order $k$ dimension $n$. For some $\lambda \in \mathbb{C}$, if the polynomial system $(\lambda \mathcal{I}-\mathcal{A}) x=0$, or equivalently $\mathcal{A} x^{k-1}=\lambda x^{[k-1]}$, has a solution $x \in \mathbb{C}^{n} \backslash\{0\}$, then $\lambda$ is called an eigenvalue of $\mathcal{A}$ and $x$ is an eigenvector of $\mathcal{A}$ associated with $\lambda$, where $x^{[k-1]}:=\left(x_{1}^{k-1}, x_{2}^{k-1}, \ldots, x_{n}^{k-1}\right)$.

In the above definition, $(\lambda, x)$ is called an eigenpair of $\mathcal{A}$. If $x$ is a real eigenvector of $\mathcal{A}$, surely the corresponding eigenvalue $\lambda$ is real. In this case, $\lambda$ is called an $H$ eigenvalue of $\mathcal{A}$. A real symmetric tensor $\mathcal{A}$ of even order $k$ is called positive semidefinite (or positive definite) if for any $x \in \mathbb{R}^{n} \backslash\{0\}, \mathcal{A} x^{k} \geq 0$ (or $\mathcal{A} x^{k}>0$ ). Denote by $\lambda_{\min }(\mathcal{A})$ the least H-eigenvalue of $\mathcal{A}$.

Lemma 2.2 (20], Theorem 5). Let $\mathcal{A}$ be a real symmetric tensor of order $k$ and dimension $n$, where $k$ is even. Then the following results hold.

(1) $\mathcal{A}$ always has $H$-eigenvalues, and $\mathcal{A}$ is positive definite (or positive semidefinite) if and only if its least $H$-eigenvalue is positive (or nonnegative).

(2) $\lambda_{\min }(\mathcal{A})=\min \left\{\mathcal{A} x^{k}: x \in \mathbb{R}^{n},\|x\|_{k}=1\right\}$, where $\|x\|_{k}=\left(\sum_{i=1}^{n}\left|x_{i}\right|^{k}\right)^{\frac{1}{k}}$. Furthermore, $x$ is an optimal solution of the above optimization if and only if it is an eigenvector of $\mathcal{A}$ associated with $\lambda_{\min }(\mathcal{A})$.

2.2. Uniform hypergraphs and eigenvalues. A hypergraph $G=(V(G), E(G))$ is a pair consisting of a vertex set $V(G)=\left\{v_{1}, v_{2}, \ldots, v_{n}\right\}$ and an edge set $E(G)=$ $\left\{e_{1}, e_{2}, \ldots, e_{m}\right\}$, where $e_{j} \subseteq V(G)$ for each $j \in[m]$. If $\left|e_{j}\right|=k$ for all $j \in[m]$, then $G$ is called a $k$-uniform hypergraph. The degree $d_{G}(v)$ or simply $d(v)$ of a vertex $v \in V(G)$ is defined as $d(v)=\left|\left\{e_{j}: v \in e_{j}\right\}\right|$. A walk in a $G$ is a sequence of alternate vertices and edges: $v_{0} e_{1} v_{1} e_{2} \ldots e_{l} v_{l}$, where $v_{i}, v_{i+1} \in e_{i}$ for $i=0,1, \ldots, l-1$. A walk is called a path if all the vertices and edges appeared on the walk are distinct. A hypergraph $G$ is called connected if every two vertices of $G$ are connected by a walk or path. 
If a hypergraph is both connected and acyclic, it is called a hypertree. The $k$-th power of a simple graph $H$, denoted by $H^{k}$, is obtained from $H$ by replacing each edge (a 2 -set) with a $k$-set by adding $(k-2)$ additional vertices [13]. The $k$-th power of a tree is called power hypertree, which is surely a $k$-uniform hypertree. In particular, the $k$-th power of a star (as a simple graph) with $m$ edges is called a hyperstar, denote by $S_{m}^{k}$. In a $k$-th power hypertree $T$, an edge is called a pendent edge of $T$ if it contains $k-1$ vertices of degree one, which are called the pendent vertices of $T$.

Lemma 2.3 (1]). If $G$ is a connected $k$-uniform hypergraph with $n$ vertices and $m$ edges, then $G$ is a hypertree if and only if $m=\frac{n-1}{k-1}$.

Definition $2.4([12)$. Let $k$ be even. A $k$-uniform hypergraph $G=(V, E)$ is called odd-bipartite, if there exists a bipartition $\left\{V_{1}, V_{2}\right\}$ of $V$ such that each edge of $G$ intersects $V_{1}$ (or $V_{2}$ ) in an odd number of vertices (such bipartition is called an odd-bipartition of $G$ ); otherwise, $G$ is called non-odd-bipartite.

The odd-bipartite hypergraphs are considered as generalizations of the ordinary bipartite graphs. The examples of non-odd-bipartite hypergraphs can been found in [14, 19. Note that the odd-bipartite hypergraphs are also called odd-transversal hypergraphs in 19. As a special case of generalized power hypergraphs defined in 14, the $k$-uniform hypergraph $G^{k, \frac{k}{2}}$ is obtained from a simple graph $G$ by blowing up each vertex into an $\frac{k}{2}$-set and preserving the adjacency relation, where $k$ is even. It was shown that $G^{k, \frac{k}{2}}$ is odd-bipartite if and only if $G$ is bipartite 14.

Let $G$ be a $k$-uniform hypergraph on $n$ vertices $v_{1}, v_{2}, \ldots, v_{n}$. The adjacency tensor of $G\left[4\right.$ is defined as $\mathcal{A}(G)=\left(a_{i_{1} i_{2} \ldots i_{k}}\right)$, an order $k$ dimensional $n$ tensor, where

$$
a_{i_{1} i_{2} \ldots i_{k}}= \begin{cases}\frac{1}{(k-1) !}, & \text { if }\left\{v_{i_{1}}, v_{i_{2}}, \ldots, v_{i_{k}}\right\} \in E(G) ; \\ 0, & \text { otherwise. }\end{cases}
$$

Observe that the adjacency tensor of a hypergraph is symmetric.

Let $x=\left(x_{1}, x_{2}, \ldots, x_{n}\right) \in \mathbb{C}^{n}$. Then $x$ can be considered as a function defined on the vertices of $G$, that is, each vertex $v_{i}$ is mapped to $x_{i}=: x_{v_{i}}$. If $x$ is an eigenvector of $\mathcal{A}(G)$, then it defines on $G$ naturally, i.e., $x_{v}$ is the entry of $x$ corresponding to $v$. If $G_{0}$ is a sub-hypergraph of $G$, denote by $\left.x\right|_{G_{0}}$ the restriction of $x$ on the vertices of $G_{0}$, or a subvector of $x$ indexed by the vertices of $G_{0}$.

Denote by $E_{G}(v)$, or simply $E(v)$, the set of edges of $G$ containing $v$. For a subset $U$ of $V(G)$, denote $x^{U}:=\Pi_{v \in U} x_{u}$. Then we have

$$
\mathcal{A}(G) x^{k}=\sum_{e \in E(G)} k x^{e}
$$

and for each $v \in V(G)$,

$$
\left(\mathcal{A}(G) x^{k-1}\right)_{v}=\sum_{e \in E(v)} x^{e \backslash\{v\}} .
$$

So the eigenvector equation $\mathcal{A}(G) x^{k-1}=\lambda x^{[k-1]}$ is equivalent to that for each $v \in V(G)$,

$$
\lambda x_{v}^{k-1}=\sum_{e \in E(v)} x^{e \backslash\{v\}} .
$$


From Lemma 2.2(2), if $k$ is even, then $\lambda_{\min }(G):=\lambda_{\min }(\mathcal{A}(G))$ can be expressed as

$$
\lambda_{\min }(G)=\min _{x \in \mathbb{R}^{n},\|x\|_{k}=1} \sum_{e \in E(G)} k x^{e} .
$$

Note than if $k$ is odd, the Eq. (2.3) does not hold. The reason is as follows. If $G$ contains at least one edge, then by Perron-Frobenius theorem, the spectral radius $\rho(\mathcal{A}(G))$ of $\mathcal{A}(G)$ is positive associated with a unit nonnegative eigenvector $x$. Now

$$
-\rho(\mathcal{A}(G)) \leq \lambda_{\min }(G) \leq \mathcal{A}(G)(-x)^{k}=-\mathcal{A}(G) x^{k}=-\rho(\mathcal{A}(G)),
$$

so $\lambda_{\min }(G)=-\rho(\mathcal{A}(G))$, which implies that $k$ is even and $G$ is odd-bipartite [21, 9, a contradiction.

Lemma 2.5. Let $G$ be a $k$-uniform hypergraph, and $(\lambda, x)$ be an eigenpair of $\mathcal{A}(G)$. If $E(u)=E(v)$ and $\lambda \neq 0$, then $x_{u}^{k}=x_{v}^{k}$.

Proof. Consider the eigenvector equation of $x$ at $u$ and $v$ respectively,

$$
\lambda x_{u}^{k}=\sum_{e \in E(u)} x^{e}, \lambda x_{v}^{k}=\sum_{e \in E(v)} x^{e} .
$$

As $E[u]=E[v], \sum_{e \in E(u)} x^{e}=\sum_{e \in E(v)} x^{e}$. The result follows.

Lemma 2.6. Let $G$ be a $k$-uniform hypergraph with at least one edge, where $k$ is even. Let $G_{0}$ be a induced sub-hypergraph of $G$. Then $\lambda_{\min }(G) \leq \lambda_{\min }\left(G_{0}\right)$. In particular, $\lambda_{\min }(G) \leq-1$.

Proof. Let $x$ be a unit first eigenvector of $G_{0}$. Define $\bar{x}$ on $G$ such that $\bar{x}(v)=x(v)$ if $v \in V\left(G_{0}\right)$ and $\bar{x}(v)=0$ otherwise. Then by Lemma 2.2.

$$
\lambda_{\min }\left(G_{0}\right)=\mathcal{A}\left(G_{0}\right) x^{k}=\mathcal{A}(G) \bar{x}^{k} \geq \min _{x \in \mathbb{R}^{n},\|x\|_{k}=1} \mathcal{A}(G) x^{k}=\lambda_{\min }(G) .
$$

If letting $G_{0}$ be an edge, then $\lambda_{\min }\left(G_{0}\right)=-1$. The result follows.

\section{Properties of the First eigenvectors}

We will give some properties of the first eigenvectors of a connected $k$-uniform $G$. We stress that $k$ is even in this and the following section.

Let $G_{1}, G_{2}$ be two vertex-disjoint hypergraphs, and let $v_{1} \in V\left(G_{1}\right), v_{2} \in V\left(G_{2}\right)$. The coalescence of $G_{1}, G_{2}$ with respect to $v_{1}, v_{2}$, denoted by $G_{1}\left(v_{1}\right) \diamond G_{2}\left(v_{2}\right)$, is obtained from $G_{1}, G_{2}$ by identifying $v_{1}$ with $v_{2}$ and forming a new vertex $u$. The graph $G_{1}\left(v_{1}\right) \diamond G_{2}\left(v_{2}\right)$ is also written as $G_{1}(u) \diamond G_{2}(u)$. If a connected hypergraph $G$ can be expressed in the form $G=G_{1}(u) \diamond G_{2}(u)$, where $G_{1}, G_{2}$ are both nontrivial and connected, then $u$ is called a cut vertex of $G$, and $G_{1}$ is called a branch of $G$ with root $u$. Clearly $G_{2}$ is also a branch of $G$ with root $u$ in the above definition.

Lemma 3.1. Let $G=G_{0}(u) \diamond H(u)$ be a connected $k$-uniform hypergraph. Let $x$ be a first eigenvector of $G$. Then the following results hold.

(1) If $H$ is odd-bipartite, then $x^{e} \leq 0$ for each $e \in E(H)$, and there exists a first eigenvector of $G$ such that it is nonnegative on one part and nonpositive on the other part for any odd-bipartition of $H$.

(2) If $x_{u}=0$, then $\sum_{e \in E_{G_{0}}(u)} x^{e \backslash\{u\}}=\sum_{e \in E_{H}(u)} x^{e \backslash\{u\}}=0$. If further $H$ is odd-bipartite, then $x^{e \backslash\{u\}}=0$ for each $e \in E_{H}(u)$. 
Proof. Let $\{U, W\}$ be an odd-bipartition of $H$, where $u \in U$. Without loss of generality, we assume that $\|x\|_{k}=1$ and $x_{u} \geq 0$. Let $\tilde{x}$ be such that

$$
\tilde{x}_{v}= \begin{cases}x_{v}, & \text { if } v \in V\left(G_{0}\right) \backslash\{u\} ; \\ \left|x_{v}\right|, & \text { if } v \in U ; \\ -\left|x_{v}\right|, & \text { if } v \in W .\end{cases}
$$

Note that $\|\tilde{x}\|_{k}=\|x\|_{k}=1$, and for each $e \in E(H), \tilde{x}^{e} \leq x^{e}$ with equality if and only if $x^{e} \leq 0$.

We prove the assertion (1) by a contradiction. Suppose that there exists an edge $e \in E(H)$ such that $x^{e}>0$. Then $\tilde{x}^{e}<x^{e}$. By Eq. (2.3), we have

$$
\lambda_{\min }(G) \leq \mathcal{A}(G) \tilde{x}^{k}<\mathcal{A}(G) x^{k}=\lambda_{\min }(G),
$$

a contradiction. So $x^{e} \leq 0$ for each $e \in E(H)$, and $\tilde{x}$ is also a first eigenvector as $\mathcal{A}(G) \tilde{x}^{k}=\mathcal{A}(G) x^{k}$. The assertion (1) follows.

For the assertion (2), let $\bar{x}$ be such that

$$
\bar{x}_{v}= \begin{cases}x_{v}, & \text { if } v \in V\left(G_{0}\right) \backslash\{u\} \\ -x_{v}, & \text { if } v \in V(H) .\end{cases}
$$

Then $\bar{x}$ is also a first eigenvector of $G$ as $\mathcal{A}(G) \bar{x}^{k}=\mathcal{A}(G) x^{k}$. Note that $x_{u}=0$ and consider the eigenvector equation Eq. (2.2) of $x$ and $\bar{x}$ at $u$, respectively.

$$
\begin{aligned}
\lambda_{\min }(G) x_{u}^{k-1}=0 & =\sum_{e \in E_{G_{0}}(u)} x^{e \backslash\{u\}}+\sum_{e \in E_{H}(u)} x^{e \backslash\{u\}}, \\
\lambda_{\min }(G) \bar{x}_{u}^{k-1}=0 & =\sum_{e \in E_{G_{0}}(u)} \bar{x}^{e \backslash\{u\}}+\sum_{e \in E_{H}(u)} \bar{x}^{e \backslash\{u\}} \\
& =\sum_{e \in E_{G_{0}}(u)} x^{e \backslash\{u\}}-\sum_{e \in E_{H}(u)} x^{e \backslash\{u\}} .
\end{aligned}
$$

Thus $\sum_{e \in E_{G_{0}}(u)} x^{e \backslash\{u\}}=\sum_{e \in E_{H}(u)} x^{e \backslash\{u\}}=0$.

If further $H$ is odd-bipartite, applying the above result to $\tilde{x}$ (also a first eigenvector of $G$ ), we have $\sum_{e \in E_{H}(u)} \tilde{x}^{e \backslash\{u\}}=0$. As $\tilde{x}^{e \backslash\{u\}} \leq 0$ for each edge $e \in E_{H}(u)$, $\tilde{x}^{e \backslash\{u\}}=0$ for each $e \in E_{H}(u)$. The assertion (2) follows by the definition of $\tilde{x}$.

Lemma 3.2. Let $G=G_{0}(u) \diamond H(u)$ be a connected $k$-uniform hypergraph. Then

$$
\lambda_{\min }\left(G_{0}\right) \geq \lambda_{\min }(G),
$$

with equality if and only if for any first eigenvector $y$ of $G_{0}, y_{u}=0$ and $\tilde{y}$ is a first eigenvector of $G$, where $\tilde{y}$ is defined by

$$
\tilde{y}_{v}= \begin{cases}y_{v}, & \text { if } v \in V\left(G_{0}\right) \\ 0, & \text { otherwise. }\end{cases}
$$

Proof. Suppose that $y$ is a first eigenvector of $G_{0},\|y\|_{k}=1$. Let $e_{0}$ be an edge of $H$ containing $u$. Define $\bar{y}$ by

$$
\bar{y}_{v}= \begin{cases}y_{v}, & \text { if } v \in V\left(G_{0}\right) ; \\ -y_{u}, & \text { if } v \in e_{0} \backslash\{u\} \\ 0, & \text { otherwise. }\end{cases}
$$


Then $\|\bar{y}\|_{k}^{k}=1+(k-1) y_{u}^{k}$, and

$$
\begin{aligned}
\mathcal{A}(G) \bar{y}^{k} & =\sum_{e \in E\left(G_{0}\right)} k \bar{y}^{e}+\sum_{e \in E(H)} k \bar{y}^{e} \\
& =\mathcal{A}\left(G_{0}\right) y^{k}-k y_{u}^{k} \\
& =\lambda_{\min }\left(G_{0}\right)-k y_{u}^{k} .
\end{aligned}
$$

By Eq. (2.3), we have

$$
\lambda_{\min }(G) \leq \frac{\mathcal{A}(G) \bar{y}^{k}}{\|\bar{y}\|_{k}^{k}}=\frac{\lambda_{\min }\left(G_{0}\right)-k y_{u}^{k}}{1+(k-1) y_{u}^{k}} \leq \lambda_{\min }\left(G_{0}\right),
$$

where the first equality holds if and only if $\bar{y}$ is also a first eigenvector of $G$, and the second equality holds if and only if $y_{u}=0$. The result now follows.

Corollary 3.3. Let $G=G_{0}(u) \diamond H(u)$ be a connected k-uniform hypergraph.

(1) If $y$ is a first eigenvector of $G_{0}$ with $y_{u} \neq 0$, then

$$
\lambda_{\min }\left(G_{0}\right)>\lambda_{\min }(G) .
$$

(2) If $x$ is a first eigenvector of $G$ such that $x_{u}=0$ and $\left.x\right|_{G_{0}} \neq 0$, then

$$
\lambda_{\min }\left(G_{0}\right)=\lambda_{\min }(G) .
$$

Proof. By Lemma 3.2 we can get the assertion (1) immediately. Let $x$ be a first eigenvector of $G$ as in (2). By Lemma 3.1 (2), $\sum_{e \in E_{G_{0}}(u)} x^{e \backslash\{u\}}=0$. Considering the eigenvector equation (2.2) of $x$ at each vertex of $V\left(G_{0}\right)$, we have

$$
\mathcal{A}\left(G_{0}\right)\left(\left.x\right|_{G_{0}}\right)^{k-1}=\lambda_{\min }(G) x^{k-1} .
$$

So $\left.x\right|_{G_{0}}$, the restriction of $x$ on $G_{0}$, is an eigenvector of $\mathcal{A}\left(G_{0}\right)$ associated with the eigenvalue $\lambda_{\min }(G)$. The result follows by Lemma 3.2.

Lemma 3.4. Let $G=G_{0}(u) \diamond H(u)$ be a connected $k$-uniform hypergraph. If $x$ is a first eigenvector of $G$, then

$$
\alpha_{H}(x):=\sum_{e \in E_{H}(u)} x^{e} \leq 0 .
$$

Furthermore, if $\alpha_{H}(x)=0$ and $\left.x\right|_{G_{0}} \neq 0$, then $x_{u}=0$ and $\lambda_{\min }\left(G_{0}\right)=\lambda_{\min }(G)$; or equivalently if $x_{u} \neq 0$, then $\alpha_{H}(x)<0$.

Proof. Let $\lambda:=\lambda_{\min }(G)$. By Eq. (2.2), for each $v \in V\left(G_{0}\right) \backslash\{u\}$,

$$
\left((\mathcal{A}(G)-\lambda \mathcal{I}) x^{k-1}\right)_{v}=\left(\left(\mathcal{A}\left(G_{0}\right)-\lambda \mathcal{I}\right)\left(\left.x\right|_{G_{0}}\right)^{k-1}\right)_{v}=0 .
$$

For the vertex $u$,

$$
\begin{aligned}
\lambda x_{u}^{k-1}=\left(\mathcal{A}(G) x^{k-1}\right)_{u} & =\sum_{e \in E_{G_{0}}(u)} x^{e \backslash\{u\}}+\sum_{e \in E_{H}(u)} x^{e \backslash\{u\}} \\
& =\left(\mathcal{A}\left(G_{0}\right)\left(\left.x\right|_{G_{0}}\right)^{k-1}\right)_{u}+\sum_{e \in E_{H}(u)} x^{e \backslash\{u\}} .
\end{aligned}
$$

So,

$$
\left(\left(\mathcal{A}\left(G_{0}\right)-\lambda \mathcal{I}\right)\left(\left.x\right|_{G_{0}}\right)^{k-1}\right)_{u}=-\sum_{e \in E_{H}(u)} x^{e \backslash\{u\}} .
$$


By Lemma 3.2 and Lemma 2.2 $(1), \mathcal{A}\left(G_{0}\right)-\lambda \mathcal{I}$ is positive semidefinite. Then $\left(\mathcal{A}\left(G_{0}\right)-\lambda \mathcal{I}\right) y^{k} \geq 0$ for any real and nonzero $y$. So, by Eq. (3.2) and Eq. (3.3), we have

$$
\begin{aligned}
0 \leq\left(\mathcal{A}\left(G_{0}\right)-\lambda \mathcal{I}\right)\left(\left.x\right|_{G_{0}}\right)^{k} & =\left(\left.x\right|_{G_{0}}\right)^{\top}\left(\left(\mathcal{A}\left(G_{0}\right)-\lambda \mathcal{I}\right)\left(\left.x\right|_{G_{0}}\right)^{k-1}\right. \\
& =-x_{u} \sum_{e \in E_{H}(u)} x^{e \backslash\{u\}} \\
& =-\alpha_{H}(x) .
\end{aligned}
$$

So we have $\alpha_{H}(x) \leq 0$.

Suppose that $\alpha_{H}(x)=0$ and $\left.x\right|_{G_{0}} \neq 0$. If $x_{u}=0$, by Corollary 3.3(2), $\lambda_{\min }\left(G_{0}\right)=\lambda_{\min }(G)$. If $x_{u} \neq 0$, then $\sum_{e \in E_{H}(u)} x^{e \backslash\{u\}}=0$. By Eq. (3.2) and Eq. (3.3), $\left(\lambda_{\min }(G),\left.x\right|_{G_{0}}\right)$ is an eigenpair of $\mathcal{A}\left(G_{0}\right)$, implying that $\lambda_{\min }(G)=\lambda_{\min }\left(G_{0}\right)$ by Lemma 3.2. However, $\left(\left.x\right|_{G_{0}}\right)_{u}=x_{u} \neq 0$, a contradiction to Corollary 3.3(1).

\section{Perturbation of the least eigenvalues}

We will give a perturbation result on the least eigenvalues under relocating a branch. Let $G_{0}, H$ be two vertex-disjoint hypergraphs, where $v_{1}, v_{2}$ are two distinct vertices of $G_{0}$, and $u$ is a vertex of $H$ (called the root of $H$ ). Let $G=G_{0}\left(v_{2}\right) \diamond H(u)$ and $\tilde{G}=G_{0}\left(v_{1}\right) \diamond H(u)$. We say that $\tilde{G}$ is obtained from $G$ by relocating $H$ rooted at $u$ from $v_{2}$ to $v_{1}$; see Fig. 4.1.
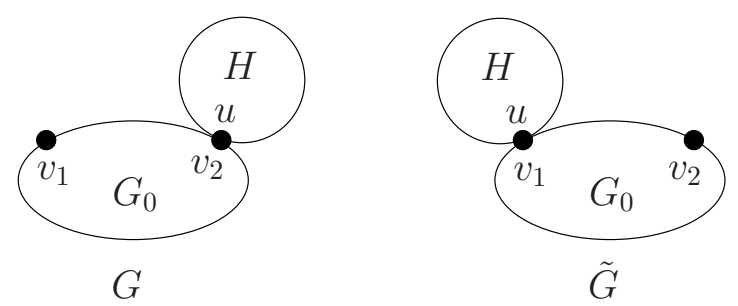

FiguRE 4.1. Relocating $H$ from $v_{2}$ to $v_{1}$

Lemma 4.1. Let $G=G_{0}\left(v_{2}\right) \diamond H(u)$ and $\tilde{G}=G_{0}\left(v_{1}\right) \diamond H(u)$ be connected $k$-uniform hypergraphs. If $x$ is a first eigenvector of $G$ such that $\left|x_{v_{1}}\right| \geq\left|x_{v_{2}}\right|$, then

$$
\lambda_{\min }(\tilde{G}) \leq \lambda_{\min }(G),
$$

with equality if and only if $x_{v_{1}}=x_{v_{2}}=0$, and $\tilde{x}$ defined in (4.5) is a first eigenvector of $\tilde{G}$.

Proof. Let $x$ be a first eigenvector of $G$ such that $\|x\|_{k}=1$ and $x_{v_{1}} \geq 0$. We divide the discussion into three cases. Denote $\lambda:=\lambda_{\min }(G)$.

Case 1: $x_{v_{2}}>0$. Write $x_{v_{1}}=\delta x_{v_{2}}$, where $\delta \geq 1$. Define $\tilde{x}$ on $\tilde{G}$ by

$$
\tilde{x}_{v}= \begin{cases}x_{v}, & \text { if } v \in V\left(G_{0}\right) ; \\ \delta x_{v}, & \text { if } v \in V(H) \backslash\{u\} .\end{cases}
$$


Then $\|\tilde{x}\|_{k}^{k}=1+\left(\delta^{k}-1\right) \sum_{v \in V(H) \backslash\{u\}} x_{v}^{k}$, and

$$
\begin{aligned}
\mathcal{A}(\tilde{G}) \tilde{x}^{k} & =\sum_{e \in E(\tilde{G})} k \tilde{x}^{e} \\
& =\mathcal{A}(G) x^{k}+\left(\delta^{k}-1\right) \sum_{e \in E(H)} k x^{e} \\
& =\lambda+\left(\delta^{k}-1\right) \sum_{e \in E(H)} k x^{e} .
\end{aligned}
$$

By the eigenvector equation of $x$ at each vertex $v \in V(H) \backslash\{u\}$,

$$
\sum_{e \in E_{H}(v)} x^{e}=\lambda x_{v}^{k}
$$

By the eigenvector equation of $x$ at $u$,

$$
\sum_{e \in E_{G}(u)} x^{e}=\alpha_{H}(x)+\gamma_{G_{0}}(x)=\lambda x_{u}^{k}
$$

where $\gamma_{G_{0}}(x):=\sum_{e \in E_{G_{0}}(u)} x^{e}$. By Eq. (4.3) and Eq. (4.4), we have

$$
\gamma_{G_{0}}(x)+\sum_{e \in E(H)} k x^{e}=\lambda \sum_{v \in V(H)} x_{v}^{k}
$$

So

$$
\begin{aligned}
\sum_{e \in E(H)} k x^{e} & =\lambda \sum_{v \in V(H)} x_{v}^{k}-\gamma_{G_{0}}(x) \\
& =\lambda \sum_{v \in V(H)} x_{v}^{k}-\left(\lambda x_{u}^{k}-\alpha_{H}(x)\right) \\
& =\lambda \sum_{v \in V(H) \backslash\{u\}} x_{v}^{k}+\alpha_{H}(x) .
\end{aligned}
$$

Thus

$$
\begin{aligned}
\mathcal{A}(\tilde{G}) \tilde{x}^{k} & =\lambda+\left(\delta^{k}-1\right)\left(\lambda \sum_{v \in V(H) \backslash\{u\}} x_{v}^{k}+\alpha_{H}(x)\right) \\
& =\lambda\left(1+\left(\delta^{k}-1\right) \sum_{v \in V(H) \backslash\{u\}} x_{v}^{k}\right)+\left(\delta^{k}-1\right) \alpha_{H}(x) \\
& =\lambda\|\tilde{x}\|_{k}^{k}+\left(\delta^{k}-1\right) \alpha_{H}(x) .
\end{aligned}
$$

As $x_{v_{2}} \neq 0, \alpha_{H}(x)<0$ by Lemma 3.4 .

$$
\lambda_{\min }(\tilde{G}) \leq \frac{\mathcal{A}(\tilde{G}) \tilde{x}^{k}}{\|\tilde{x}\|_{k}^{k}}=\lambda+\frac{\left(\delta^{k}-1\right) \alpha_{H}(x)}{\|\tilde{x}\|_{k}^{k}} \leq \lambda=\lambda_{\min }(G),
$$

where the first equality holds if and only if $\tilde{x}$ is a first eigenvector of $\tilde{G}$, and the second equality holds if and only if $\delta=1$, i.e. $x_{v_{1}}=x_{v_{2}}$. So, the equality holds if and only if $x_{v_{1}}=x_{v_{2}}$ and $\tilde{x}$ is a first eigenvector of $\tilde{G}$. By the eigenvector equations of $x$ and $\tilde{x}$ at $v_{2}$ respectively, we will get $\alpha_{H}(x)=0$, a contradiction. So, in this case, $\lambda_{\min }(\tilde{G})<\lambda_{\min }(G)$. 
Case 2: $x_{v_{2}}=0$. First assume $x_{v_{1}}=0$. Define $\tilde{x}$ on $\tilde{G}$ by

$$
\tilde{x}_{v}= \begin{cases}x_{v}, & \text { if } v \in V\left(G_{0}\right) ; \\ x_{v}, & \text { if } v \in V(H) \backslash\{u\} .\end{cases}
$$

Then $\|\tilde{x}\|_{k}^{k}=1$, and

$$
\lambda_{\min }(\tilde{G}) \leq \mathcal{A}(\tilde{G}) \tilde{x}^{k}=\mathcal{A}(G) x^{k}=\lambda_{\min }(G),
$$

with equality if and only if $\tilde{x}$ is a first eigenvector of $\tilde{G}$.

Now assume that $x_{v_{1}}>0$. By Corollary 3.3 (2) and its proof, $\lambda_{\min }(G)=\lambda_{\min }\left(G_{0}\right)$ as $x_{u}=0$ and $\left.x\right|_{G_{0}} \neq 0$; furthermore, $\left.x\right|_{G_{0}}$ is a first eigenvector of $G_{0}$. By Corollary 3.3 $(1), \lambda_{\min }\left(G_{0}\right)>\lambda_{\min }(\tilde{G})$ as $\left(\left.x\right|_{G_{0}}\right)_{v_{1}} \neq 0$, thinking of $v_{1}$ a coalescence vertex between $G_{0}$ and $H$ in $\tilde{G}$. So $\lambda_{\min }(\tilde{G})<\lambda_{\min }(G)$.

Case 3: $x_{v_{2}}<0$. Write $x_{v_{1}}=-\delta x_{v_{2}}$, where $\delta \geq 1$. Define $\tilde{x}$ on $\tilde{G}$ by

$$
\tilde{x}_{v}= \begin{cases}x_{v}, & \text { if } v \in V\left(G_{0}\right) ; \\ -\delta x_{v}, & \text { if } v \in V(H) \backslash\{u\} .\end{cases}
$$

By a similar discussion to Case 1 by replacing $\delta$ by $-\delta$, we also have $\lambda_{\min }(\tilde{G})<$ $\lambda_{\min }(G)$.

Denoted by $\mathcal{T}_{m}\left(G_{0}\right)$ the class of hypergraphs with each obtained from a fixed connected hypergraph $G_{0}$ by attaching some hypertrees at some vertices of $G_{0}$ respectively (i.e. identifying a vertex of a hypertree with some vertex of $G_{0}$ each time) such that the number of its edges equals $\varepsilon\left(G_{0}\right)+m$. A hypergraph is called a minimizing hypergraph in a certain class of hypergraphs if its least eigenvalue attains the minimum among all hypergraphs in the class.

We will characterize the minimizing hypergraph(s) in $\mathcal{T}_{m}\left(G_{0}\right)$. Denote by $G=$ $G_{0}(u) \diamond S_{m}^{k}(u)$ the coalescence of $G_{0}$ and $S_{m}^{k}$ by identifying one vertex of $G_{0}$ and the central vertex of $S_{m}^{k}$ and forming a new vertex $u$.

Theorem 4.2. Let $G_{0}$ be a connected $k$-uniform hypergraph. If $G$ is a minimizing hypergraph in $\mathcal{T}_{m}\left(G_{0}\right)$, then $G=G_{0}(u) \diamond S_{m}^{k}(u)$ for a unique vertex $u$ of $G_{0}$.

Proof. Suppose that $G$ is a minimizing hypergraph in $\mathcal{T}_{m}\left(G_{0}\right)$, and $G$ has no the structure as desired in the theorem. We will get a contradiction by the following three cases.

Case 1: $G$ contains hypertrees attached at two or more vertices of $G_{0}$. Let $T_{1}, T_{2}$ be two hypertrees attached at $v_{1}, v_{2}$ of $G_{0}$ respectively. Let $x$ be a first eigenvector of $G$. Assume $\left|x_{v_{1}}\right| \geq\left|x_{v_{2}}\right|$. Relocating $T_{2}$ rooted at $v_{2}$ and attaching to $v_{1}$, we will get a hypergraph $\bar{G} \in \mathcal{T}_{m}\left(G_{0}\right)$ such that $\lambda_{\min }(\bar{G}) \leq \lambda_{\min }(G)$ by Lemma 4.1 Repeating the above operation, we finally arrive at a hypergraph $G^{(1)}$ with only one hypertree $T^{(1)}$ attached at one vertex $u_{0}$ of $G_{0}$ such that $\lambda_{\min }\left(G^{(1)}\right) \leq \lambda_{\min }(G)$.

Case 2: $T^{(1)}$ contains edges with three or more vertices of degree greater than one, i.e. $T^{(1)}$ is not a power hypertree. Let $e$ be one of such edges containing $u, v, w$ with $d(u), d(v), d(w)$ all greater than one. Let $x$ be a first eigenvector of $G^{(1)}$, and assume that $\left|x_{u}\right| \geq\left|x_{w}\right|$. Relocating the hypertree rooted at $w$ and attaching to $u$, we will get a hypergraph $\hat{G} \in \mathcal{T}_{m}\left(G_{0}\right)$ such that $\lambda_{\min }(\hat{G}) \leq \lambda_{\min }\left(G^{(1)}\right)$ by Lemma 4.1. Repeating the above operation on the edge $e$ until $e$ contains exactly 2 vertices of degree greater than one, and on each other edges like $e$, we finally arrive at a 
hypergraph $G^{(2)}$ such that the unique hypertree $T^{(2)}$ attached at $u_{0}$ is a power hypertree, and $\lambda_{\min }\left(G^{(2)}\right) \leq \lambda_{\min }\left(G^{(1)}\right)$.

Case 3: $T^{(2)}$ contains pendent edges not attached at $u_{0}$. Let $x$ be a first eigenvector of $G^{(2)}$. We assert that $\left|x_{u_{0}}\right|=\max _{v \in V\left(G_{0}\right)}\left|x_{v}\right|$. Otherwise, there exists a vertex $v_{0}$ of $G_{0}$ such that $\left|x_{v_{0}}\right|>\left|x_{u_{0}}\right|$. Relocating $T^{(2)}$ rooted at $u_{0}$ and attaching to $v_{0}$, we will get a hypergraph $\tilde{G} \in \mathcal{T}_{m}\left(G_{0}\right)$ such that $\lambda_{\min }(\tilde{G})<\lambda_{\min }\left(G^{(2)}\right)$ by Lemma 4.1. Then $\lambda_{\min }(\tilde{G})<\lambda_{\min }(G)$, a contradiction to $G$ being minimizing.

We also assert that $\left|x_{u_{0}}\right|=\max _{v \in V\left(G^{(2)}\right)}\left|x_{v}\right|$. Otherwise, let $w_{0} \in V\left(T^{(2)}\right) \backslash\left\{u_{0}\right\}$ such that $\left|x_{w_{0}}\right|=\max _{v \in V\left(G^{(2)}\right)}\left|x_{v}\right|>\left|x_{u_{0}}\right|$. Then $w_{0}$ must have degree greater than one; otherwise letting $e$ be the only edge containing $w_{0}$, and letting $\bar{w}_{0}$ a vertex in $e$ with degree greater than one, relocating the branch attached at $\bar{w}_{0}$ and attaching to $w_{0}$, we will get a hypergraph with smaller least eigenvalue by Lemma 4.1. a contradiction. Now relocating $G_{0}$ rooted at $u_{0}$ and attaching to $w_{0}$, we also get a hypergraph with smaller least eigenvalue by Lemma 4.1. By a similar discussion, $u_{0}$ is the unique vertex satisfying $\left|x_{u_{0}}\right|=\max _{v \in V\left(G^{(2)}\right)}\left|x_{v}\right|$.

Finally let $e$ be a pendent edge of $T^{(2)}$ attached at $v \neq u_{0}$. Relocating $e$ from $v$ to $u_{0}$, we get a hypergraph with smaller least eigenvalue by Lemma 4.1, a contradiction. The result now follows.

If taking $G_{0}$ to be a single edge in Theorem 4.2, then we get the following corollary immediately.

Corollary 4.3. Among all k-uniform hypertree with $m$ edges, the minimizing hypergraph is unique, namely the hyperstar $S_{m}^{k}$.

It is easy to verify that a hypertree $T$ is odd-bipartite (by induction on the number of edges). So the spectrum of $\mathcal{A}(T)$ is symmetric with respect to the origin 21, and hence $\lambda_{\min }(T)=-\rho(T)$. Therefore, Corollary 4.3 implies a result of [16] when $k$ is even, which says that $S_{m}^{k}$ is the unique hypergraph with maximum spectral radius among all $k$-uniform hypertrees with $m$ edges.

Next we consider the case that $G_{0}$ is non-odd-bipartite. Let $C_{2 n+1}$ be an odd cycle of length $2 n+1$ (as a simple graph). Let $k \geq 4$ be a positive even integer. Then $C_{2 n+1}^{k, \frac{k}{2}}$ is a non-odd-bipartite $k$-uniform hypergraph [14. Let $K_{n}^{k}$ be a complete $k$-uniform hypergraph on $n \geq k+1$ vertices. It is easy to verify that $K_{n}^{k}$ is nonodd-bipartite. If taking $G_{0}$ be $C_{2 n+1}^{k, \frac{k}{2}}$ or $K_{n}^{k}$ in Theorem 4.2, we get the following results immediately.

Corollary 4.4. The minimizing hypergraph in $\mathcal{T}_{m}\left(C_{2 n+1}^{k, \frac{k}{2}}\right)$ is the unique hypergraph $C_{2 n+1}^{k, \frac{k}{2}}(u) \diamond S_{m}^{k}(u)$ for an arbitrary vertex u of $C_{2 n+1}^{k, \frac{k}{2}}$, up to isomorphism.

Corollary 4.5. The minimizing hypergraph in $\mathcal{T}_{m}\left(K_{n}^{k}\right)$ is the unique hypergraph $K_{n}^{k}(u) \diamond S_{m}^{k}(u)$ for an arbitrary vertex $u$ of $K_{n}^{k}$, up to isomorphism.

\section{REFERENCES}

1. C. Berge, Hypergraphs: Combinatorics of finite sets, North-Holland, 1989.

2. K. C. Chang, K. Pearson, T. Zhang, Perron-Frobenius theorem for nonnegative tensors, Commu. Math. Sci., 6(2008), 507-520.

3. K. Chang, L. Qi, T. Zhang, A survey on the spectral theory of nonnegative tensors, Numer. Linear Algebra Appl., 20 (2013), 891-912. 
4. J. Cooper, A. Dutle, Spectra of uniform hypergraphs, Linear Algebra Appl., 436(9)(2012), 3268-3292.

5. Y.-Z. Fan, Y.-H. Bao, T. Huang, Eigenvariety of nonnegative symmetric weakly irreducible tensors associated with spectral radius and its application to hypergraphs, Linear Algebra Appl., 564 (2019), 72-94.

6. Y.-Z. Fan, T. Huang, Y.-H. Bao, C.-L. Zhuan-Sun, Y.-P. Li, The spectral symmetry of weakly irreducible nonnegative tensors and connected hypergraphs, Trans. Amer. Math. Soc., 372(3) (2019), 2213-2233.

7. Y.-Z. Fan, M. Khan, Y.-Y. Tan, The largest H-eigenvalue and spectral radius of Laplaican tensor of non-odd-bipartite generalized power hypergraphs, Linear Algebra Appl., 504(2016), 487-502.

8. Y.-Z. Fan, Y.-Y. Tan, X.-X. Peng, A.-H. Liu, Maximizing spectral radii of uniform hypergraphs with few edges, Discuss. Math. Graph Theory, 36(2016), 845-856

9. Y.-Z. Fan, Y. Wang, Y.-H. Bao, J.-C. Wan, M. Li, Z. Zhu, Eigenvectors of Laplacian or signless Laplacian of hypergraphs associated with zero eigenvalue, Linear Algebra Appl., 2019, 579: 244-261.

10. Y.-Z. Fan, Y. Wang, Y.-B. Gao, Minimizing the least eigenvalues of unicyclic graphs with application to spectral spread, Linear Algebra Appl., 429 (2008), 577-588.

11. S. Friedland, S. Gaubert, L. Han, Perron-Frobenius theorem for nonnegative multilinear forms and extensions, Linear Algebra Appl., 438(2013), 738-749.

12. S. Hu, L. Qi, The eigenvectors associated with the zero eigenvalues of the Laplacian and signless Laplacian tensors of a uniform hypergraph, Discrete Appl. Math., 169(2014), 140151.

13. S. Hu, L. Qi, J. Y. Shao, Cored hypergraphs, power hypergraphs and their Laplacian Heigenvalues, Linear Algebra Appl., 439 (2013), 2980-2998.

14. M. Khan, Y.-Z. Fan, On the spectral radius of a class of non-odd-bipartite even uniform hypergraphs, Linear Algebra Appl., 480(2015), 93-106.

15. M. Khan, Y.-Z. Fan, Y.-Y. Tan, The H-spectra of a class of generalized power hypergraphs, Discrete Math., 339(2016), 1682-1689.

16. H. Li, J.-Y. Shao, L. Qi, The extremal spectral radii of k-uniform supertrees, J. Comb. Optim., 32(2016), 741-764.

17. L.-H. Lim, Singular values and eigenvalues of tensors: a variational approach, Proceedings of the 1st IEEE International Workshop on Computational Advances in Multi-Sensor Adaptive Processing, 2005, pp. 129-132.

18. L. Lu, S. Man, Connected hypergraphs with small spectral radius, Linear Algebra Appl., $\mathbf{5 0 9}(2016), 206-227$.

19. V. Nikiforov, Hypergraphs and hypermatrices with symmetric spectrum, Linear Algebra Appl., $\mathbf{5 1 9}(2017), 1-18$.

20. L. Qi, Eigenvalues of a real supersymmetric tensor, J. Symbolic Comput., 40(6)(2005), 13021324 .

21. J.-Y. Shao, H.-Y. Shan and B.-F. Wu, Some spectral properties and characterizations of connected odd-bipartite uniform hypergraphs, Linear Multilinear Algebra, 63(2015), 23592372 .

22. Y. Yang and Q. Yang, Further results for Perron-Frobenius theorem for nonnegative tensors, SIAM J Matrix Anal. Appl., 31(5)(2010), 2517-2530.

23. Y. Yang and Q. Yang, Further results for Perron-Frobenius theorem for nonnegative tensors II, SIAM J Matrix Anal. Appl., 32(4)(2011), 1236-1250.

24. Y. Yang, Q. Yang, On some properties of nonnegative weakly irreducible tensors, Available at arXiv: $1111.0713 \mathrm{v} 2$.

School of Mathematical Sciences, Anhui University, Hefei 230601, P. R. China

E-mail address: fanyz@ahu.edu.cn

School of Mathematical Sciences, Anhui University, Hefei 230601, P. R. China

E-mail address: $2937242741 @ q q . c o m$

School of Mathematical Sciences, Anhui University, Hefei 230601, P. R. China

E-mail address: wangy@ahu.edu.cn 